\title{
How to surpass the deep-sea glass sponges mechanically
}

\author{
Quan-Wei $\mathrm{Li}^{1}$ and Bo-Hua Sun*1 \\ ${ }^{1}{ }^{1}$ School of Civil Engineering 83 Institute of Mechanics and Technology, \\ Xi'an University of Architecture and Technology, Xi'an 710055, China. \\ Corresponding author: Bo-Hua Sun, e-mail: sunbohua@xauat.cn, http://imt.xauat.edu.cn
}

\begin{abstract}
The biomimetic design of engineering structures is based on biological structures with excellent mechanical properties, which are the result of billions of years of evolution. However, current biomimetic structures, such as ordered lattice materials, are still inferior to many biological materials in terms of structural complexity and mechanical properties. For example, the structure of Euplectella aspergillum, a type of deep-sea glass sponge, is an eye-catching source of inspiration for biomimetic design; however, guided by scientific theory, how to engineer structures surpassing the mechanical properties of E. aspergillum remains an unsolved problem. The lattice structure of the skeleton of E. aspergillum consists of vertically, horizontally, and diagonally oriented struts, which provide superior strength and flexural resistance compared with the conventional square lattice structure. Herein, the structure of E. aspergillum was investigated in detail, and by using the theory of elasticity, a lattice structure inspired by the bionic structure was proposed. The mechanical properties of the sponge-inspired lattice structure surpassed the sponge structure under a variety of loading conditions, and the excellent performance of this configuration was verified experimentally. The proposed lattice structure can greatly improve the mechanical properties of engineering structures, and it improves strength without much redundancy of material. This study achieved the first surpassing of the mechanical properties of an existing sponge-mimicking design. This design can be applied to lattice structures, truss systems, and metamaterial cells.
\end{abstract}

Keywords: Bioinspired materials, Lattice structure, Euplectella aspergillum, Buckling, Optimization

\section{INTRODUCTION}

Over billions of years of evolution, some lightweight, high-strength, and tough biological structural material$\mathrm{s}$, such as shells and bones, have appeared in nature. Compared with synthetic materials, the performance of biological materials is often superior, with better compromises between different properties. Due to the constraints of their growth environment, all living things have had to evolve very selective biomaterials and structures with impressive mechanical properties [1-4]. The application of mechanics and materials science method$\mathrm{s}$ has promoted the understanding of biomaterials and guided the design of biomimetic structures [5-8]. The cage-like skeletons of Euplectella aspergillum, a type of deep-sea glass sponge, are able to resist the effects of strong hydrostatic pressure, currents, and attacks from other marine organisms in the deep sea [9]. Hydrodynam- ic studies have shown that this skeletal structure helps to reduce seawater resistance and improve mechanical stability [10]. The skeleton is tubular overall and squaregridded locally, consisting of bundles of vertically, horizontally, and diagonally arranged needle-like struts, with the diagonal struts covering the square grid in a staggered tessellated fashion (Figure 1c). This double-diagonal reinforced square grid structure has stronger mechanical properties than existing engineered structures [11], and the diagonal support allows the square grid skeleton to withstand bending, shear, and torsional loads [9, 12, 13].

The development of lattice materials (particularly bio-inspired lattice materials) is based on a desire to design multifunctional materials and structures. Highporosity lattice materials are inherently light, and have large specific stiffness and specific strength due to the directional distribution of stress [13, 14]. Manufacturing lightweight and high-strength materials is a major 


\section{a}

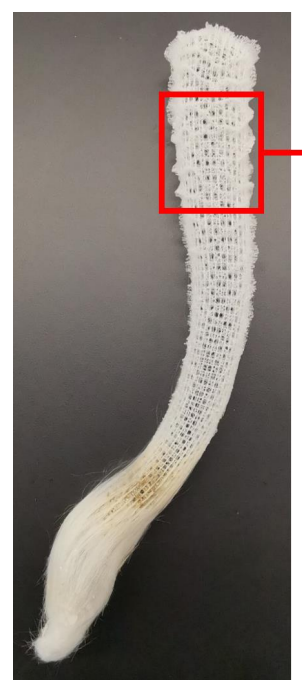

b

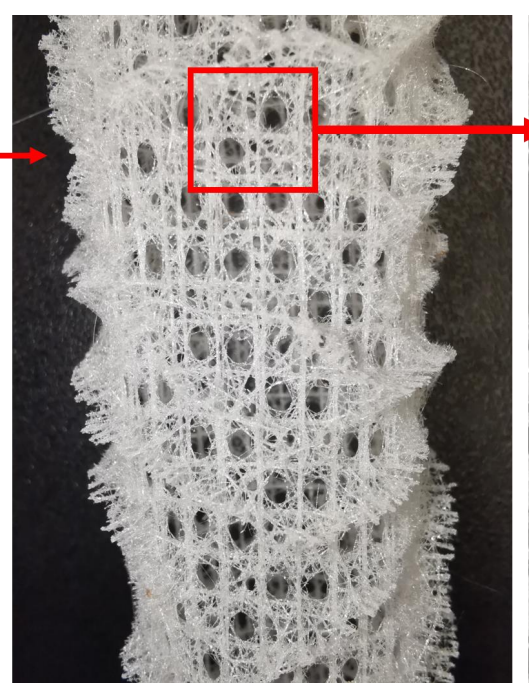

c

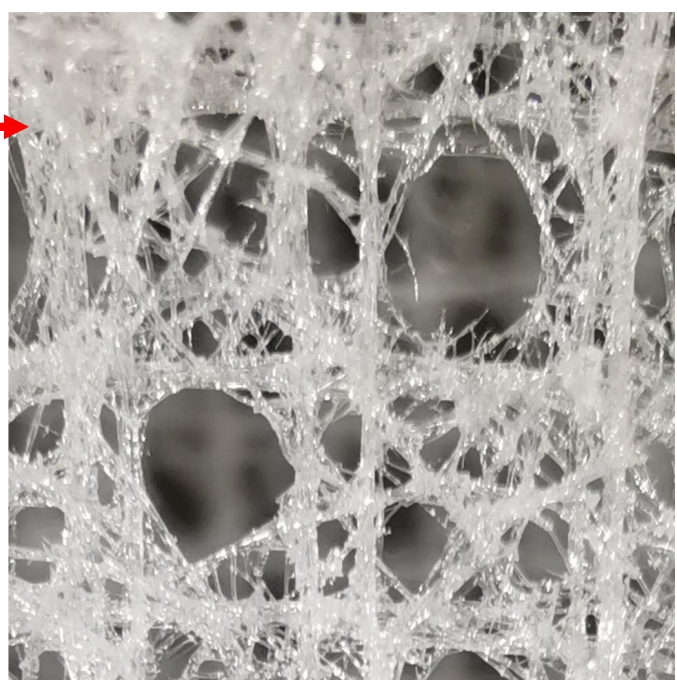

FIG. 1: Skeleton structure of Euplectella aspergillum. (a) The whole skeleton structure of E. aspergillum. (b) Lattice-like organization. (c) Chessboard-like grid alternately arranged.

goal in the field of engineering. Compared with ordinary man-made materials, many biomaterials have very high strength, yet remain lightweight and porous [15]. Various geometric arrays of porous grid-like periodic structured biomedical implants [16] have been manufactured. Lightweight lattice structures can also be the core of sandwich structures, which has advantages in multifunctional structural applications in the aerospace, automotive, and civil engineering industries [17]. Truss lattice materials are often used for energy absorption, especially in the design of impact and explosion-resistant structures [18]. Sandwich structures with a lattice or foam core have better performance than the same quality compact structure. A hinge design in a lattice can consume energy through buckling [19-22]. The excellent mechanical properties of the skeleton of E. aspergillum have been extensively studied [23, 24]. However, most studies have focused on the performance of sponge spicules [25-28]. The shape of the sponge skeleton has been applied to the appearance of buildings [29]. To date, only Matheus et al. [11] have carried out research on the diagonally reinforced square grid unit structure of the sponge skeleton, and the optimized configuration is very close to the spongemimicking design. However, the potential of this square grid form in mechanics has not been fully explored, and the key scientific problem of surpassing the performance of the sponge structure has not been solved. In this study, our objective was to develop a sponge-inspired structure with beter mechanical properties than the skeleton of $E$. aspergillum.

Biological systems usually face limited resources, and the skeleton is designed to provide structural stability at minimal cost, which is the common theme of various biological evolutions. The evolutionary trends in natural biomaterials share many of the same aspirations as the development of engineered materials, such as weight reduction while ensuring effective strength and rigidity [30], as seen in feathers [31], plant stems [32], and porcupines quills [33]. Numerous biological materials are inspiring to scientists and engineers. The experience and knowledge gained from research into the structure of biological materials can guide the design of new bionic structures. Lattice materials with complex topologies can be used in a wide variety of engineering applications, such as tower structures, lightweight sandwich structures, and filters [13, 34].

Biological structures have been optimized over longterm evolution and have hierarchical structures [35]. Regarding the optimization of biological structures in nature, the optimal shape will be different for different load patterns. Using optimal design to discover new unit geometry is an effective way to further improve engineered 
structures [36]. The concept of structural optimization design reflects the best use of materials and resources.

The optimization goal of this study for the proposed sponge-inspired structure was to maximize the load-bearing capacity of the structure under differen$\mathrm{t}$ loads while using the least material possible. First, we carried out finite element simulations of different lattice structures (including the sponge-mimicking lattice structure developed in [11]), calculated the responses of the structure under various load conditions, and verified the experimental and finite element results of [11]. Then, based on our findings, we developed a sponge-inspired design surpassing the mechanical properties of the sponge, which can be used to guide the cell design of lattice structures and metamaterials. It can also be applied to the design of large man-made structures, such as space grids, towers, and bridges.

\section{FINITE ELEMENT SIMULATIONS}

\subsection{Underlying assumptions}

In this study, we were primarily concerned with the in-plane performance of the lattice structure of the skeleton of E. aspergillum. Detailed measurements on digital photos of glass sponges had previously been carried out in [11]. In order to design the sponge-mimicking configuration as simply as possible, Matheus et al. established the following basic framework of assumptions: the in-plane geometry is uniform, and all elements have the same shape in the thickness direction; all diagonal elements have the same in-plane dimension; all non-diagonal elements have the same in-plane dimension; and in the volume calculations, the area where the overlapping beams cross is negligible. We also adopted these assumptions in this study. The cross-section of the sponge-mimicking design from [11] (Design A) is shown in Figure 2(a), with the non-diagonal elements in black and the diagonal elements in green. Figure 2(b)-(d) are the cross-sections of Designs B, C, and D, respectively, which are common engineering structures designed in [11]. Different configurations have equal volume, and the volume ratio of nondiagonal elements to diagonal elements stays the same
(Design D has only non-diagonal elements, so the total volume stays the same).

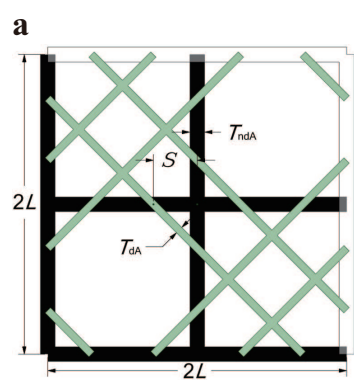

b
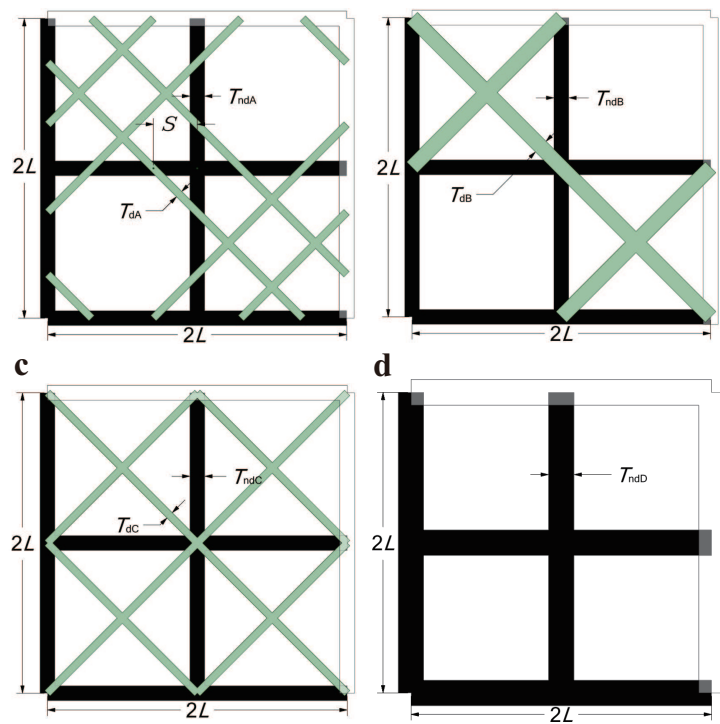

d

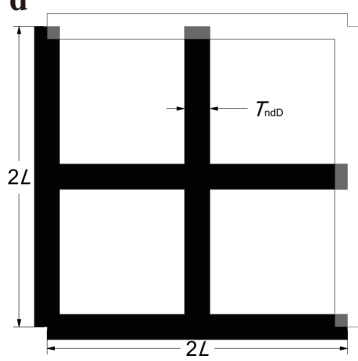

FIG. 2: Schematic diagrams of unit cross-section designs (designed by [11]). (a) Design A (sponge-mimicking design); (b) Design B; (c) Design C; (d) Design D. In the diagrams, $T_{\mathrm{ndA}}=T_{\mathrm{ndB}}=T_{\mathrm{ndC}}=0.1 L, T_{\mathrm{dA}}=\frac{1}{2} T_{\mathrm{dB}}=T_{\mathrm{dC}}=$ $0.05 \mathrm{~L}, T_{\mathrm{ndD}}=0.1 L\left(1+\frac{1}{\sqrt{2}}\right), S=\frac{L}{\sqrt{2}+2}$.

\subsection{Finite element results and analysis}

In order to verify the excellent mechanical properties of Design A (sponge-mimicking design), we conducted finite element simulations using Ansys Workbench and compared the results with the simulation and test result$\mathrm{s}$ in [11]. The geometries were constructed using twodimensional solid elements, and we captured the material's response using an incompressible Neo-Hookean material model with a shear modulus of $\mu=14.5 \mathrm{MPa}$. Figure 3(a) shows the comparison of the uniaxial compression results of a $6 \times 6$ square grid, Figure $3(\mathrm{~b})$ shows the comparison of the three-point bending results of an $11 \times 2$ square grid, and Figure 3(c) shows the comparison of a $20 \times 20$ grid for the variation of critical buckling stress results with angle. It can be seen from Figure 3 that our simulation results are in good agreement with the experimental and simulation results in [11] (the stress contours of uniaxial compression and three-point bending 
are shown in Supplementary Figure 1 and Supplementary Figure 2). The sponge-mimicking design (Design A) has the highest load-bearing capacity and the highest critical buckling stress.
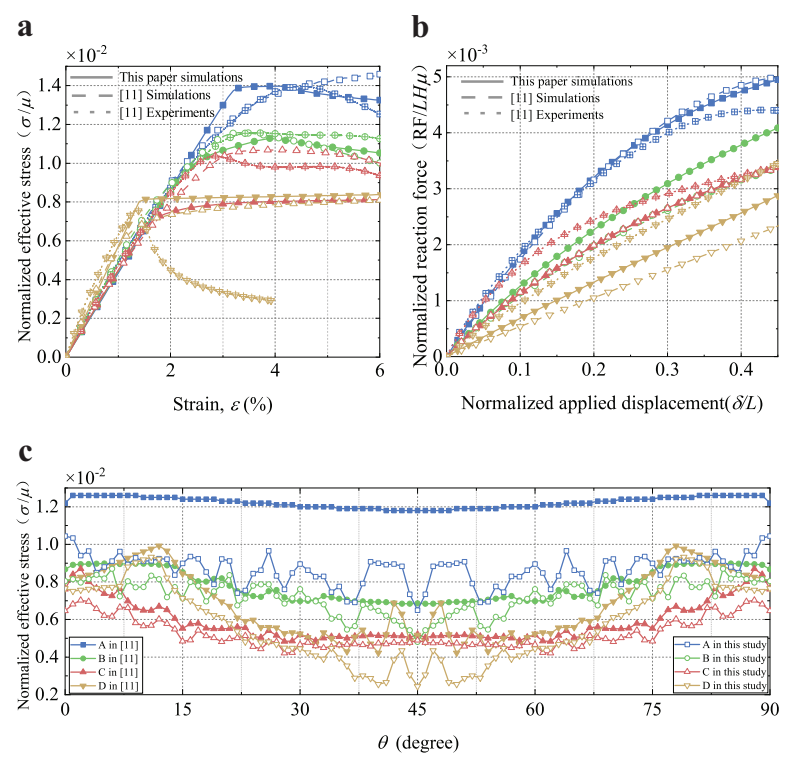

FIG. 3: Simulations in this study compared with those in [11]. (a) Comparison results of uniaxial compression. (b) Comparison results of $11 \times 2$ square grid three-point bending. (c) Comparison results of $20 \times 20$ grid for the variation of critical buckling stress with angle.

\subsection{Simulations of various loads}

The excellent performance of Design A under uniaxial compression and three-point bending had been verified, but the load conditions of the glass sponge in the submarine environment are far more complicated than the simulated load conditions. In order to verify that the superior mechanical properties of Design A are not limited to a few simple load conditions, we conducted finite element simulation to study the performance of Design A under a wider variety of load conditions.

First, we carried out steady-state thermal analysis and modal analysis for the four designs, and the results are shown in Figure 4. The thickness of the sample was $40 \mathrm{~mm}$, the Young's modulus was $200 \mathrm{MPa}$, and the Poisson's ratio was 0.3 . In the steady-state thermal analysis, the specimens were fixedly supported at the top and bot- tom ends, the material model had a thermal expansion coefficient of $1.2 \mathrm{E}-5 /{ }^{\circ} \mathrm{C}$, and the temperature rose from $22^{\circ} \mathrm{C}$ to $100^{\circ} \mathrm{C}$. We recorded the reaction force generated by the steady-state thermal effect. The reaction force of sponge-mimicking design (Design A) had the lowest support reaction force under the same heating conditions. In the modal analysis, specimens were fixedly supported at the bottom ends, and we found that the natural frequency of Design A was second highest after Design C. This preliminarily indicated that Design A has excellent thermal stability and dynamic stability.

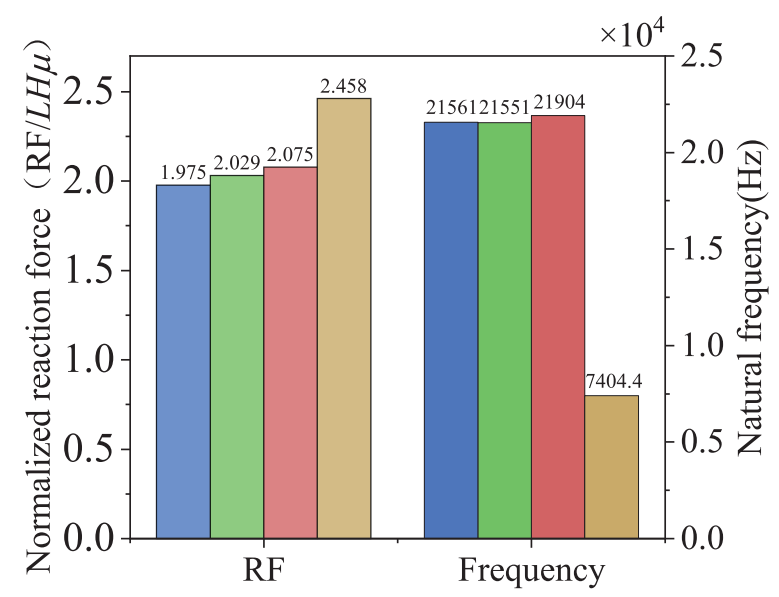

FIG. 4: Results of steady-state thermal analysis and modal analysis.

Next we performed simulations on four designs of 6 $\times 6$ square grid elements under shear, bending, torsion, and tensile loading conditions. We captured the material's response using an incompressible Neo-Hookean material model with a shear modulus of $\mu=14.5 \mathrm{MPa}$, and the loading schematic and simulation results are shown in Figure 5 (the stress contour diagrams of the various load situations are shown in supplementary Figures 3-6).

It can be seen from Figure 5(b) that Design D had low shear stiffness and poor shear resistance. The initial shear stiffnesses of Designs A, B, and C were almost equal, and their shear stiffness decreased at different displacements. Design A had the best shear resistance. Under in-plane bending load, we selected the cross-section thickness of $2.5 \mathrm{~mm}$ for simulation, as shown in Figure 5(c). According to Figure 5(d), the bending stiffness of Design D was the highest, and the initial bending stiff- 
a

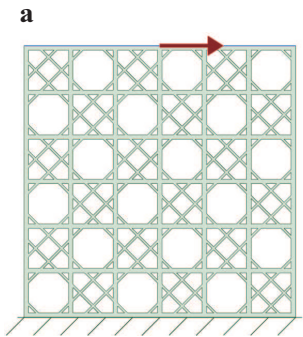

b

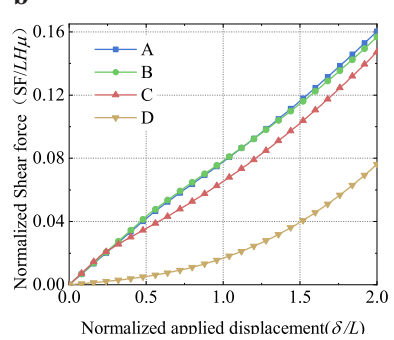

c
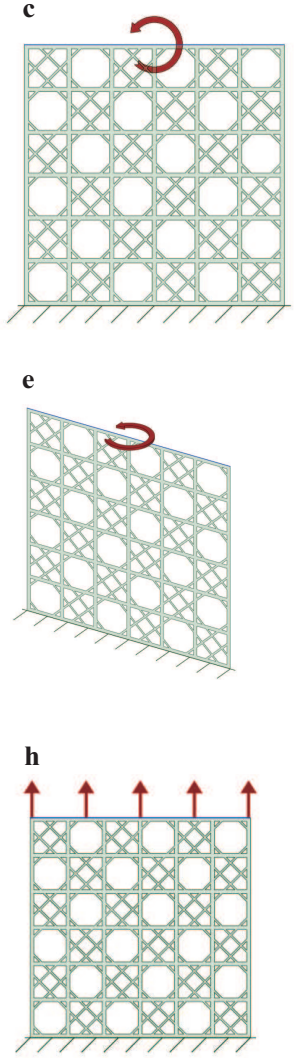

d

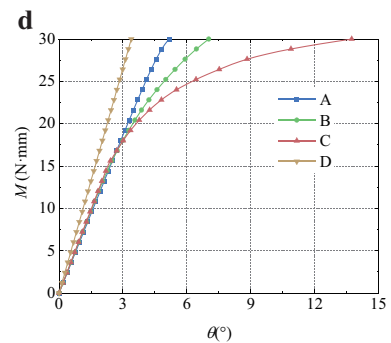

f

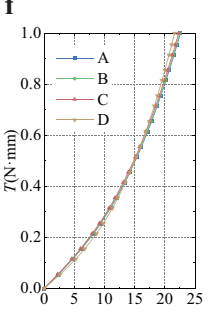

$\theta\left({ }^{\circ}\right)$

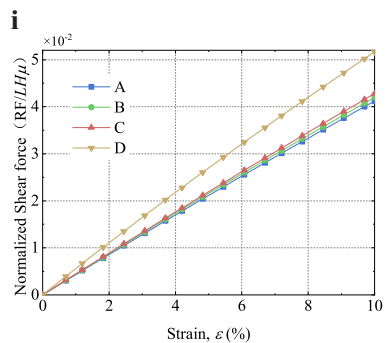

FIG. 5: Simulation results of $6 \times 6$ square grid elements under shear, bending, torsion, and tension load. (a) Shear load. (b) Shear simulation results. (c) Bending load. (d) Bending simulation results. (e) Torsion load. (f) Torsion simulation results (thickness $1 \mathrm{~mm}$ ). (g) Torsion simulation results (thickness $2.5 \mathrm{~mm}$ ). (h) Tensile load. (i) Tension simulation results.

nesses of Designs A, B, and C were basically equal. With the increase of the rotation angle $\theta$, the bending stiffnesses of Designs B and C decreased, and the bending resistance of Design A was second only to Design D. Under torsional load, we selected the section thicknesses of 1 $\mathrm{mm}$ and $2.5 \mathrm{~mm}$ for simulation. As shown in Figure 5(f), when the thickness was $1 \mathrm{~mm}$, the designs showed relatively similar responses. The initial torsional stiffness of Design D was slightly lower, and with the continuous loading of the corner, the torsional bearing capacity exceeded that of other designs. As shown in Figure $5(\mathrm{~g})$, when the thickness was $2.5 \mathrm{~mm}$, Designs A, B, and $\mathrm{C}$ showed almost the same response, characterized by strong torsion resistance, whereas Design D had lower torsional stiffness and poor torsion resistance. According to Figure 5(i), under uniaxial tensile load, the performance was completely opposite to uniaxial compression. The tensile performances of Designs A, B, and C were similar, although the performance of Design A was the worst since it had the lowest tensile stiffness. Design D had the best tensile performance. Under tension load, local trusses are not easily affected by buckling caused by compression load, so they exhibit the opposite phenomenon of uniaxial compression.

Design A performed well under uniaxial compression and three-point bending steady-state thermal load, its thermal stability and shear resistance were significantly better than other designs, and its dynamic stability, bending performance, and torsion resistance were also very good. Design A (the sponge-mimicking design) was weaker than Design D (the square grid without diagonal design) only under uniaxial tension. This reflects the fact that the sponge skeleton has evolved in an outstanding way over hundreds of millions of years in the natural environment to resist various loads. Inspired by the sponge grid configuration, we attempted to design a grid configuration with even better performance under various load conditions.

\section{BEYOND THE SPONGE-MIMICKING DESIGN}

Only when the most basic scientific principles of biological structures are understood can we design materials transcending their performance. The most basic scientific principle of biological structures is the theory of elasticity. Euler's formula $F_{c r}=\frac{\pi^{2} E I}{l^{2}}$, which is part of the theory of elasticity, reveals an important law. To increase the buckling critical force $F_{c r}$ under equal mass, the trusses must be thicker (the moment of inertia $I$ is greater) and shorter (the length $l$ is smaller), as shown in Figure 6(a). Figure 6(b) and Figure 6(c) indicate that the stiffness of 
a thicker and shorter beam is greater under both bending load and torsion load.

Inspired by the sponge skeleton and the design rule, we developed Design E, which is shown in Figure 7(a). The non-diagonal elements were identical to Design A (the sponge-mimicking design). However, the diagonal elements were twice as thick compared to Design A, but the total volume was kept equal to that of Design A. We numerically simulated the uniaxial compression and shear performance of the $6 \times 6$ square grid cell of Design $\mathrm{E}$ and compared the results with the results of Designs A-D. As shown in Figure 7(b), Design E had the lowest initial compression stiffness but the highest load-bearing capacity at strain 6\%. As shown in Figure 7(c), the shear resistance of Design E was significantly weaker than Designs A, B, and C. The compressive bearing capacity of Design E was close to that of Design A, but due to the lack of penetration of the diagonal elements, the shear resistance was significantly weakened.

The comparison from Designs A-E indicated the enhancement of the diagonal connection is necessary, which confirmed the conclusion of [13] that diagonal support is essential for the skeleton to withstand bending, shear, and torsion loads. Matheus et al. optimized Design A, and obtained a configuration with better compressive performance. Design A and its optimization results are very close (the critical buckling stress of the optimization result is $9.55 \%$ higher than that of Design A). After carefully studying the optimization problem of Design A under the number of multiple diagonals, Matheus et al. concluded that the double-diagonal sponge design has the best compressive performance [11].

Does Design A and its optimization results represen$\mathrm{t}$ the limit of such a square lattice structure? Has this double-diagonal-enhanced square grid design reached its optimal performance? Developing a design surpassing the performance of Design A has become a world problem. We have the courage to challenge and devote ourselves to solving this problem, and finally try to surpass Design A under multi-load conditions. There are three main problems with the work of Matheus et al. [11]. First, it ignored the volume distribution in the horizontal and vertical directions in non-diagonal elements. Sec- ond, the design with a single diagonal number (Design B) still had room for optimization, and the performance had the potential to surpass Design A. Third, it set a single optimization goal (that is, the highest critical buckling stress), but the resulting configuration may not be able to achieve excellent comprehensive performance under multi-load conditions.

The problem that needs to be solved is clear: how can a new configuration be developed that fully exceeds Design A under multi-load conditions? In this part of the study, our goal was to create a lattice structure design surpassing Design A under multiple load conditions. To this end, we developed an idea of optimizing the design on the basis of Design E (diagonal elements have a smaller length-to-thickness ratio, which means they are thicker and shorter, but the diagonal elements do not have a through connection) and Design B. The schematic diagram of the optimization parameters in a $6 \times 6$ square grid is shown in Figure 8.

In the process of simulating lattice structure, material nonlinearity and geometric nonlinearity are big challenges for convergence and computing, resulting in unbearable consumption of computational resources and time to find the peak load. Therefore, we assumed that the magnitude of the initial stiffness corresponds to the strength of the load-bearing capacity so that the optimization results could be obtained quickly. In order to obtain an excellent configuration under multi-load conditions at the lowest cost, we selected the optimization objectives to be the compression, shear loads under a given small displacement, and the rotation angle under a given small bending moment. We selected three parameters to be optimized: the volume ratio of non-diagonal elements to diagonal elements $\lambda_{1}, \lambda_{1}=\frac{T_{\mathrm{v}}+T_{\mathrm{h}}}{\sqrt{2} T_{\mathrm{d}}}$, the ratio of the volume of the vertical element to the horizontal element $\lambda_{2}, \lambda_{2}=\frac{T_{\mathrm{v}}}{T_{\mathrm{h}}}$, and the distance between the diagonal element and the square grid node $\Delta L$. On the basis of Matheus et al.'s assumption framework [11], we kept the total volume constant (equal material consumption) and adjusted the volume distribution of horizontal and vertical elements to study the performance of the $6 \times 6$ square grid structure. We set the optimization objectives as the responses of the structure under compression, shear, 
a

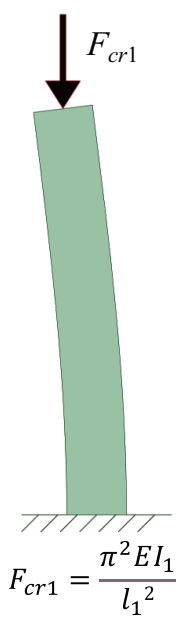

b

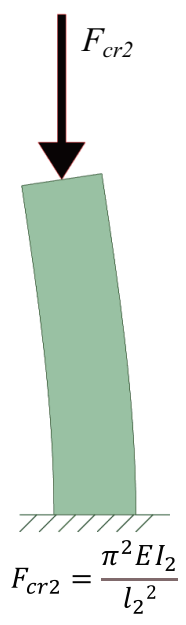

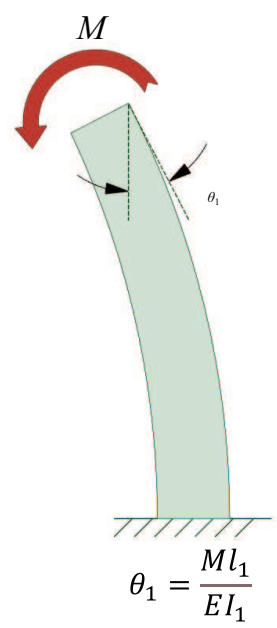

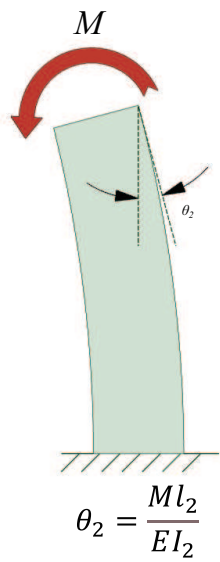

c

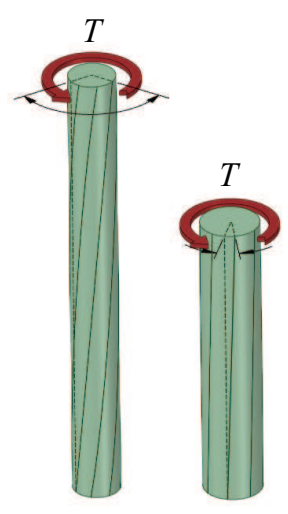

$\varphi_{1}=\frac{T l_{1}}{G I_{p 1}} \quad \varphi_{2}=\frac{T l_{2}}{G I_{p 2}}$

FIG. 6: Design rule of equal mass struts. (a) Considering the stability under compression, $I_{1}<I_{2}, l_{1}>l_{2}$, so $F_{c r 1}<F_{c r 2}$. (b) Under moment load, $I_{1}<I_{2}, l_{1}>l_{2}$, so $\theta_{1}>\theta_{2}$. (c) Under torsion load, $I_{p 1}<I_{p 2}, l_{1}>l_{2}$, so $\varphi_{1}>\varphi_{2}$.
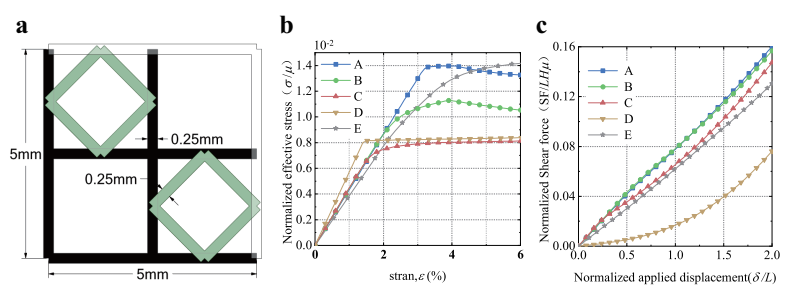

FIG. 7: Design E and its simulation results compared with Designs A-D. (a) Cell of Design E. (b) Uniaxial compression simulation results. (c) Shear simulation results.

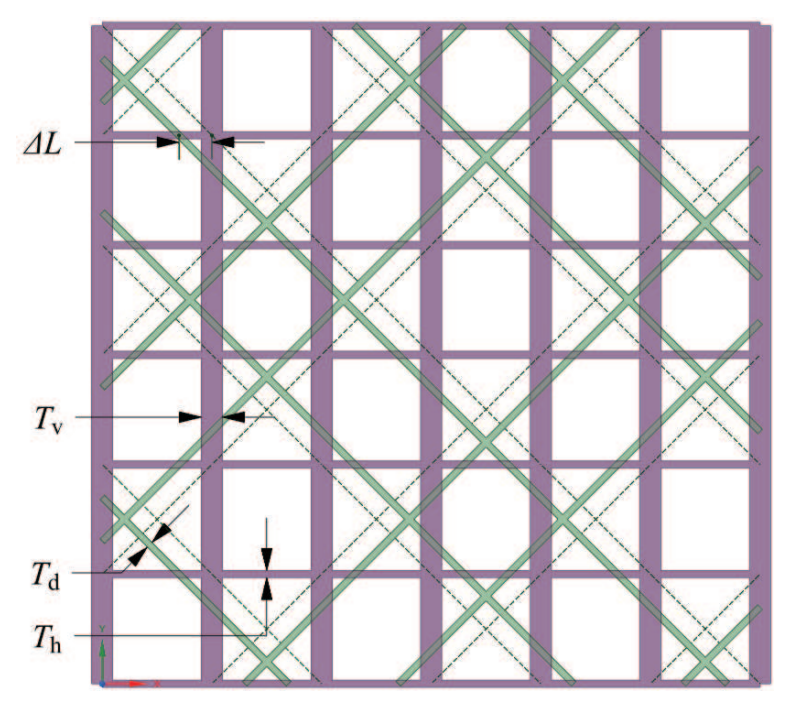

FIG. 8: Optimization design parameters. and bending loads, with the aim of obtaining configurations where the three optimization objectives all exceed Design A (the sponge-mimicking design. We focused optimization analysis on the in-plane performance of the grid structure, taking a thickness of $1000 \mathrm{~mm}$, and using an incompressible Neo-Hookean model with a shear modulus of 14.5 MPa.

The multi-objective optimization problem can be expressed as:

$$
\begin{aligned}
& \text { Find : }\left[\lambda_{1}, \lambda_{2}, \Delta L\right] . \\
& \text { Max : } F_{c}, F_{s} \\
& \text { Min : } \theta \\
& \text { s.t. }\left\{\begin{array}{l}
F_{c} \geq 825 \mathrm{~N} \\
F_{s} \geq 450 \mathrm{~N} \\
\theta \leq 0.04^{\circ}
\end{array}\right.
\end{aligned}
$$

where $F_{c}$ is the axial force when the compressive strain is $1 \%, F_{s}$ is the shear force when the displacement is 0.5 $\mathrm{mm}$, and $\theta$ is the angle under the $100 \mathrm{~N} \cdot \mathrm{mm}$ bending moment.

We adopted the multi-objective genetic algorithm (MOGA) provided in Ansys for multi-objective optimization, and obtained the following optimization design results: $\lambda_{1}=1.55, \lambda_{2}=2.963$, and $\Delta L=0.32081$. We denoted the corresponding design as Design F.

Matheus et al. [11] took the highest critical buckling 
stress as the optimization goal and performed seven separate optimizations on the diagonally reinforced square grid structure. The optimal result, which we herein denote as A-Opti1, was in the form of a double diagonal and only differed from Design $\mathrm{A}$ in the values of two parameters: the volume ratio of non-diagonal elements to diagonal elements $\lambda$ and the node distance $S$. For AOpti1, $\lambda=0.9, S=0.6788$, and for Design A, $\lambda=\sqrt{2}$, $S=\frac{2.5}{\sqrt{2}+2}$, where $\lambda=\frac{T_{\mathrm{ndA}}}{\sqrt{2} T_{\mathrm{dA}}}$, as shown in Figure 2(a). In order to compare with the optimal results of the sponge configuration and maintain the consistency of the simulations in this paper, we used Ansys to optimize Design A with the optimization goal of high buckling resistance, and we denoted the optimal result as A-Opti2 $(\lambda=0.74914, S=0.76853)$.

Next, we performed finite element simulation of the sponge-mimicking design (Design A) and its optimization results (A-Opti1 and A-Opti2) under various load conditions. The results are shown in Figure 9 (the stress contour diagram under each load situation is shown in supplementary Figures 7-12). We set the response of Design A as 1, and the excellent performance of Design F can be clearly seen in Table 1 . Under uniaxial compression, three-point bending, uniaxial tension, torsion, and bending loads, the performance of Design F was optimal. Under shear load, although the initial stiffness of Design $\mathrm{F}$ was low, with the increase of displacement, its shear bearing capacity exceeded that of Design A. In terms of thermal stability and dynamic stability, Design F exceeded Designs A, A-Opti1, and A-Opti2. This indicates that the superior mechanical properties of Design $\mathrm{F}$ are not limited to individual loading conditions. We believe Design F comprehensively surpasses the mechanical performance of Design A.

It is important to note that the optimal design results presented in this paper have preconditions, that is, the comprehensive optimal value of multi-objective optimization under certain basic assumptions and given constraints. The result of the optimal design is not unique. We can change the constraint conditions according to the structural performance requirements so as to obtain the optimal design under specific conditions, and to realize the programmability of the mechanical properties of the
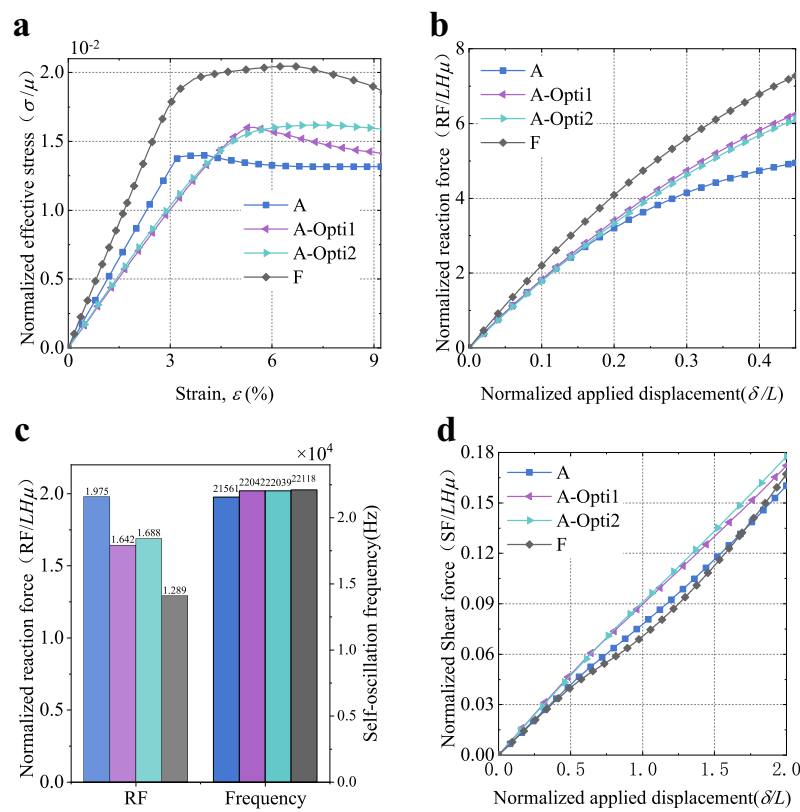

d
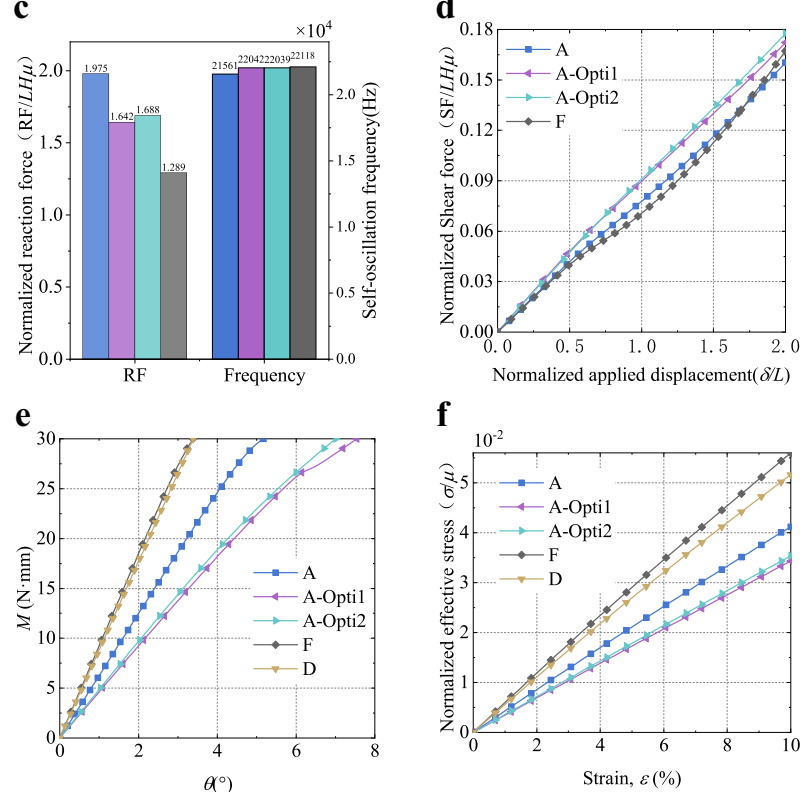

g

h
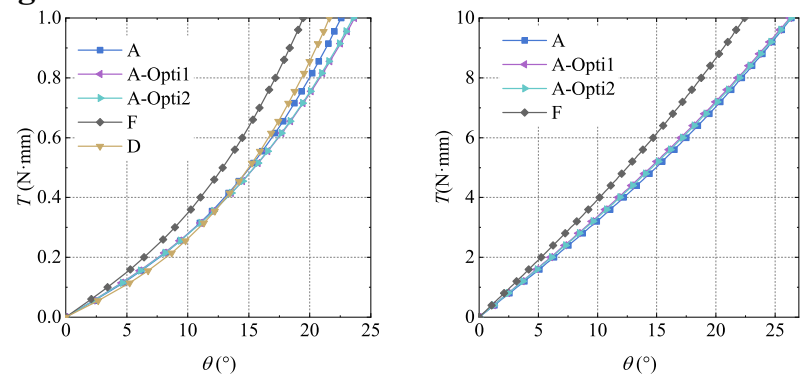

FIG. 9: Comparison of simulation results between Design $\mathbf{F}$ and sponge configuration (Designs A, A-Opti1, and A-Opti2) under different load conditions. (a) Uniaxial compression simulation results. (b) Three-point bending simulation results. (c) Thermal and dynamic stability simulation results. (d) Shear simulation results. (e) Bending simulation results. (f) Uniaxial tension simulation results. (g) Torsion simulation results (thickness $1 \mathrm{~mm}$ ). (h) Torsion simulation results (thickness $2.5 \mathrm{~mm}$ ). 
lattice structure.

TABLE I: Comparison of performance under each load. From top to bottom: uniaxial compression load at a strain of $6 \%$, load at a deflection of $1.125 \mathrm{~mm}$, reaction force after warming, natural frequency, shear force at a displacement of $2.5 \mathrm{~mm}$, angle of rotation at a moment of $30 \mathrm{~N} \cdot \mathrm{mm}$, uniaxial tension load at a strain of $10 \%$, angle of rotation of $1 \mathrm{~mm}$ thick specimens at a torque of $1 \mathrm{~N} \cdot \mathrm{mm}$. The responses of Design A were set as 1, and the surpassing percentage of Design $\mathrm{F}$ is shown as below.

\begin{tabular}{l|cccc}
\hline Load & A & A-Opti1 & $\mathrm{F}$ & Surpassing percentage \\
\hline Uniaxial compression & 1 & 1.181 & 1.538 & $53.8 \%$ \\
Three-point bending & 1 & 1.261 & 1.471 & $47.1 \%$ \\
Thermal stability & 1 & 0.831 & 0.653 & $53.1 \%$ \\
Natural frequency & 1 & 1.022 & 1.026 & $2.6 \%$ \\
Shearing & 1 & 1.074 & 1.043 & $4.3 \%$ \\
Bending & 1 & 1.460 & 0.654 & $52.9 \%$ \\
Uniaxial tension & 1 & 0.833 & 1.358 & $35.8 \%$ \\
Torsion & 1 & 1.050 & 0.863 & $15.9 \%$ \\
\hline
\end{tabular}

\section{APPLICATION AND EXPERIMENTAL VERIFICATION}

Our design results are not limited to the current sponge grid scale, but can be extended to the design of structures on a wide range of length scales. In the analysis of [11], we did not take the influence of gravity into account, which may become an important source of load for large structures. Some extreme applications in engineering, such as increasing the length of aircraft wingspan without increasing the mass, increasing the overhanging distance of large buildings, may pose great challenges to the design of the structures. Considering the application in large structures, we designed a large cantilever beam of size $6 \mathrm{~m} \times 36 \mathrm{~m}$ by creating a $4 \times 24$ square grid. We then subjected the beam to finite element analysis to compare the deflection under gravity of Design A (sponge-mimicking design), Design $\mathrm{C}$ (common design in engineering), Design F (optimized design in this study), and Design G (common design in engineering) at equal volumes (without increasing mass). The loading diagram is shown in Figure 9. The left end was fixed, and the structure was subjected to self-weight only. The thick- ness of the specimen was taken as $6 \mathrm{~m}$, with a density of $7850 \mathrm{~kg} / \mathrm{m}^{3}$, the Young's modulus was $200 \mathrm{MPa}$, and the Poisson's ratio was 0.3 . The result of the longitudinal displacement contour image magnified by 100 times is shown in Figure 10 (the stress contour diagram is shown in Supplementary Figure 13).

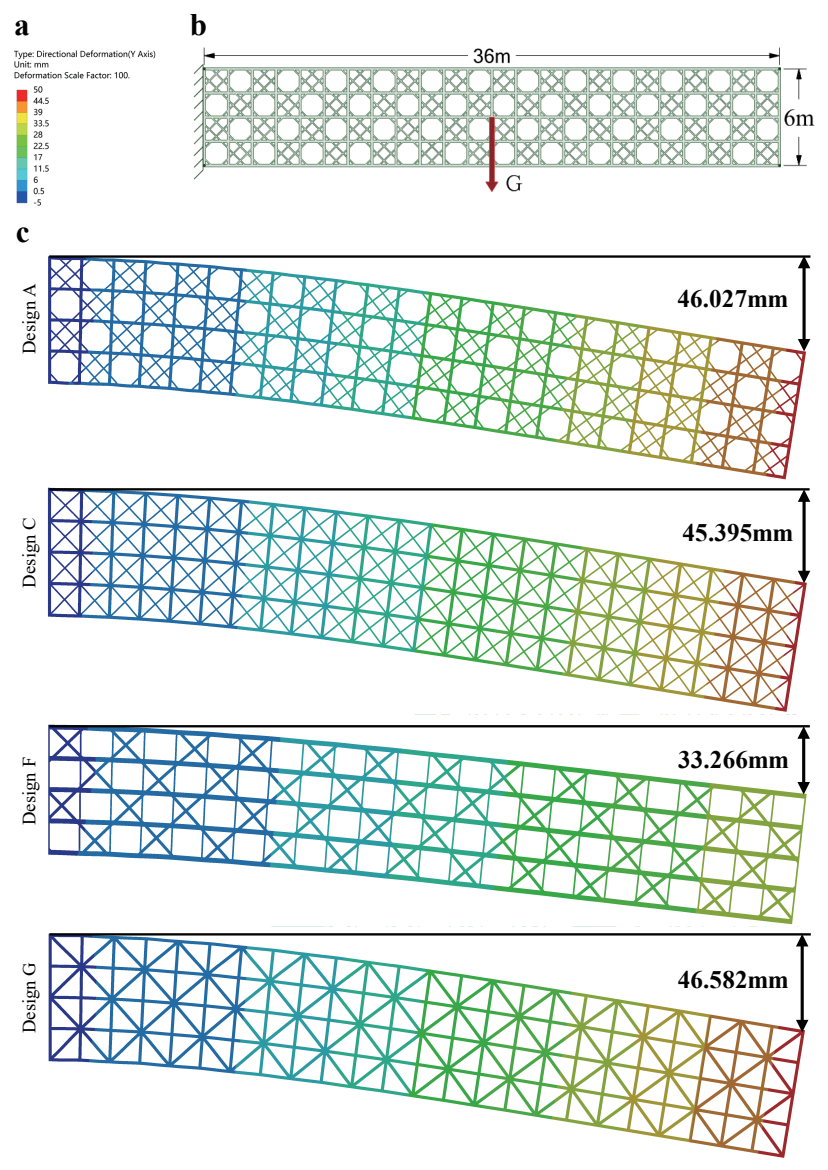

FIG. 10: Longitudinal displacement contour diagram under self-weight of a large cantilever beam. (a) Longitudinal displacement contour diagram legend. (b) Cantilever beam with the size of $6 \mathrm{~m} \times 36 \mathrm{~m}$. (c) Displacement contour diagram of Design A (sponge-mimicking design), Design C (common design in engineering), Design F (optimized design proposed in this study) and Design G (common design in engineering).

The maximum vertical deflection of Designs A, C, $\mathrm{F}$, and $\mathrm{G}$ were $46.027 \mathrm{~mm}, 45.395 \mathrm{~mm}, 33.266 \mathrm{~mm}$, and $46.582 \mathrm{~mm}$, respectively. Under equal mass, Design $\mathrm{F}$ had the best deflection resistance, and the deflection was reduced by $27.73 \%$ compared with Design A. Therefore, Design $\mathrm{F}$ has huge potential for application to super large 
cantilever structures. In order to further verify the superiority of the performance of Design F over Design A, we carried out an experiment of uniaxial compression performance, and the results are shown in Figure 11. It is evident that the uniaxial compressive load carrying capacity and stiffness of Design $\mathrm{F}$ were stronger than those of Design A. Due to the poor quality between layers of $3 \mathrm{D}$ printed specimens, there were some defects visible to the naked eye, and the serious ones are shown in Figure 11(c). After considering the larger initial defect (1 $\mathrm{mm})$, the experiment data were still slightly lower than the finite element simulation results, and the laws and trends obtained by the experiment were consistent with the finite element simulation results.
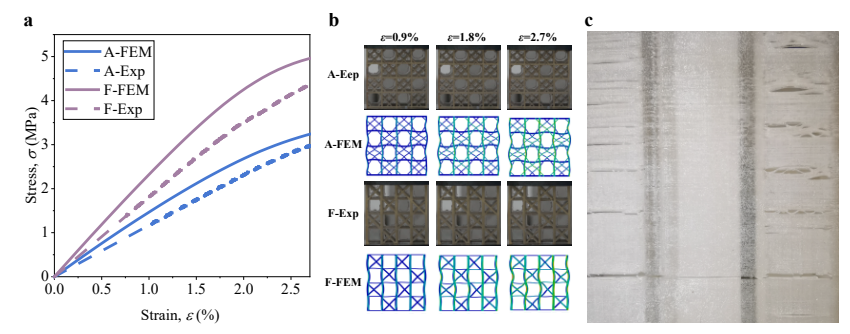

FIG. 11: Finite element and experiment results of proposed design (Design F) and sponge-mimicking design (Design A). (a) Comparison of finite element and experiment data. (b) Finite element deformation images and experiment photos. (c) Defect morphology of the specimen surface where the thickness is small.

\section{CONCLUSION}

Inspired by the deep-sea glass sponge structure, we proposed a new lattice design configuration that surpasses the sponge-mimicking design from a previous study under various load conditions. First we verified the excellent mechanical properties of the sponge-mimicking design. Then we optimized the grid structure to obtain a new configuration with superior performance under multi-load conditions. Next, we verified the superior applicability of our configuration for large cantilever beam structures using finite element method. Finally, specimens with the sponge-mimicking design and the new configuration were $3 \mathrm{D}$ printed to demonstrate the surpassing over the sponge design by uniaxial compression experiments. Our proposed design can be used to guide the cell design of lattice structures and metamaterials. Its engineering applications range from various industrial and civil infrastructures to micro-nano-level structural elements.

The evolution of nature and the depth of its scientific laws are amazing to contemplate. We would hazard a guess that for E. aspergillum, the optimized results we achieved may be one of the paths of its future evolution.

[1] W. Huang et al., Multiscale Toughening Mechanisms in Biological Materials and Bioinspired Designs. Adv Mater 31, e1901561 (2019).

[2] G. Mayer, Rigid biological systems as models for synthetic composites. Science 310, 1144-1147 (2005).

[3] P. Y. Chen et al., Structure and mechanical properties of selected biological materials. J Mech Behav Biomed Mater 1, 208-226 (2008).

[4] R. O. Ritchie, The conflicts between strength and toughness. Nat Mater 10, 817-822 (2011).

[5] E. Munch et al., Tough, bio-inspired hybrid materials. Science 322, 1516-1520 (2008).

[6] M. E. Launey et al., Designing highly toughened hybrid composites through nature-inspired hierarchical complexity. Acta Materialia 57, 2919-2932 (2009).

[7] M. M. Porter, J. McKittrick, M. A. Meyers, Biomimetic Materials by Freeze Casting. Jom 65, 720-727 (2013).

[8] M. A. Meyers, J. McKittrick, P. Y. Chen, Structural Biological Materials: Critical Mechanics-Materials Connections. Science 339, 773-779 (2013).

[9] J. Aizenberg et al., Skeleton of Euplectella sp.: structural hierarchy from the nanoscale to the macroscale. Science 309, 275-278 (2005).

[10] G. Falcucci et al., Extreme flow simulations reveal skeletal adaptations of deep-sea sponges. Nature 595, 537-541 (2021).

[11] M. C. Fernandes, J. Aizenberg, J. C. Weaver, K. Bertoldi, Mechanically robust lattices inspired by deep-sea glass sponges. Nat Mater 20, 237-241 (2021).

[12] V. Deshpande, M. Ashby, N. Fleck, Foam topology: bending versus stretching dominated architectures. Acta materialia 49, 1035-1040 (2001).

[13] A. S. Phani, M. I. Hussein, Dynamics of lattice materials. (Wiley Online Library, 2017).

[14] S. E. Naleway, M. M. Porter, J. McKittrick, M. A. Meyers, Structural Design Elements in Biological Materials: 
Application to Bioinspiration. Adv Mater 27, 5455-5476 (2015).

[15] D. Jang, L. R. Meza, F. Greer, J. R. Greer, Fabrication and deformation of three-dimensional hollow ceramic nanostructures. Nat Mater 12, 893-898 (2013).

[16] L. E. Murr et al., Next-generation biomedical implants using additive manufacturing of complex, cellular and functional mesh arrays. Philos Trans A Math Phys Eng Sci 368, 1999-2032 (2010).

[17] N. A. Fleck, V. S. Deshpande, M. F. Ashby, Microarchitectured materials: past, present and future. Proceedings of the Royal Society A: Mathematical, Physical and Engineering Sciences 466, 2495-2516 (2010).

[18] J. J. Harrigan, S. R. Reid, A. Seyed Yaghoubi, The correct analysis of shocks in a cellular material. International Journal of Impact Engineering 37, 918-927 (2010).

[19] T. Frenzel, C. Findeisen, M. Kadic, P. Gumbsch, M. Wegener, 417-127Tailored Buckling Microlattices as Reusable Light-Weight Shock Absorbers. Adv Mater 28, 5865-5870 (2016).

[20] B. Haghpanah, L. Salari-Sharif, P. Pourrajab, J. Hopkins, L. Valdevit, Multistable Shape-Reconfigurable Architected Materials. Adv Mater 28, 7915-7920 (2016).

[21] D. Restrepo, N. D. Mankame, P. D. Zavattieri, Phase transforming cellular materials. Extreme Mechanics Letters 4, 52-60 (2015).

[22] S. Shan et al., Multistable Architected Materials for Trapping Elastic Strain Energy. Adv Mater 27, 4296-4301 (2015).

[23] J. C. Weaver et al., Hierarchical assembly of the siliceous skeletal lattice of the hexactinellid sponge Euplectella aspergillum. J Struct Biol 158, 93-106 (2007).

[24] J. C. Weaver et al., Unifying Design Strategies in Demosponge and Hexactinellid Skeletal Systems. The Journal of Adhesion 86, 72-95 (2010).

[25] A. Woesz et al., Micromechanical properties of biological silica in skeletons of deep-sea sponges. Journal of Materials Research 21, 2068-2078 (2006).
[26] S. L. Walter, B. D. Flinn, G. Mayer, Mechanisms of toughening of a natural rigid composite. Materials Science and Engineering: C 27, 570-574 (2007).

[27] M. A. Monn, J. C. Weaver, T. Zhang, J. Aizenberg, H. Kesari, New functional insights into the internal architecture of the laminated anchor spicules of Euplectella aspergillum. Proc Natl Acad Sci U S A 112, 4976-4981 (2015).

[28] M. A. Monn, K. Vijaykumar, S. Kochiyama, H. Kesari, Lamellar architectures in stiff biomaterials may not always be templates for enhancing toughness in composites. Nat Commun 11, 373 (2020).

[29] A. du Plessis et al., Biomimicry for 3D concrete printing: A review and perspective. Additive Manufacturing 38, (2021).

[30] A. Velasco-Hogan, J. Xu, M. A. Meyers, Additive Manufacturing as a Method to Design and Optimize Bioinspired Structures. Adv Mater 30, e1800940 (2018).

[31] T. N. Sullivan et al., A lightweight, biological structure with tailored stiffness: The feather vane. Acta Biomater 41, 27-39 (2016).

[32] L. J. Gibson, Biomechanics of cellular solids. J Biomech 38, 377-399 (2005).

[33] W. Yang, C. Chao, J. McKittrick, Axial compression of a hollow cylinder filled with foam: a study of porcupine quills. Acta Biomater 9, 5297-5304 (2013).

[34] K. Li, P. E. Seiler, V. S. Deshpande, N. A. Fleck, Regulation of notch sensitivity of lattice materials by strut topology. International Journal of Mechanical Sciences 192, (2021).

[35] M. Meenakshi Sundaram, G. K. Ananthasuresh, Gustave Eiffel and his optimal structures. Resonance 14, 849-865 (2009).

[36] A. G. Evans, J. W. Hutchinson, N. A. Fleck, M. F. Ashby, H. N. G. Wadley, The topological design of multifunctional cellular metals. Progress in Materials Science 46, 309-327 (2001). 